\title{
Point-spread function stability of the SNAP telescope
}

\author{
M.J. Sholl ${ }^{1}$, R. Besuner ${ }^{2}$, P. Jelinsky ${ }^{1}$, M.L. Lampton ${ }^{1}$, \\ ${ }^{1}$ University of California at Berkeley \\ ${ }^{2}$ Robert Besuner Consulting, Madera, CA
}

\begin{abstract}
SNAP is a proposed space-based experiment designed to study dark energy and alternate explanations of the acceleration of the universe's expansion by performing a series of complementary systematic-controlled astrophysical measurements. The principal mission activities are the construction of an accurate Type Ia supernova Hubble diagram (the supernova program), and conducting a wide-area weak gravitational lensing (WL) survey. WL measurements benefit from a highly constant point spread function (PSF). The goal of this study is to quantify the anticipated variations in PSF arising from on-orbit thermal variations and shrinkage associated with dryout of the composite telescope metering structure. A combined thermo-mechanical-optical analysis tool was developed, and WL metrics whisker, ellipticity and effective anisotropy quantified for thermal and composite structure dryout effects. Stability limits necessary for WL are defined, and compared to stability tolerances defined for the supernova program. The mission is designed for operations at at the Earth-Sun L2 Lagrange point, where thermal disturbances from Earth are minimal. In this study, the effects of seasonal variations in solar flux, transients introduced when pointing the body-fixed Ka-band antenna toward Earth and $90^{\circ}$ roll maneuvers (planned every three months of operations) are quantified, and introduced into the optical system. Vector whisker and effective anisotropy change rates were computed, and found to be well below the WL requirement for stability. The effects of composite structure shrinkage due to on-orbit $\mathrm{H}_{2} \mathrm{O}$ desorption are discussed, and estimated to be significant, but symmetric and predictable during the supernova/deep WL phase of the mission (3-21 months after launch). When the dedicated WL phase of the mission begins (21 months after launch), structural dryout will be largely complete, with predicted daily changes well within WL limts.
\end{abstract}

Keywords: three-mirror telescopes, space astronomy, dark energy, wide-field imaging, weak lensing, anisotropy Contact: sholl@ssl.berkeley.edu

\section{SNAP OBSERVATORY OVERVIEW}

The science objectives of the SNAP mission ${ }^{1}$ are twofold: a deep repeated photometric survey of supernovae, and a wide-field survey of weak gravitational lensing (WL) of distant galaxies. This second objective requires the accurate determination of apparent shapes of hundreds of millions of galaxies. The instrumental signature imposed by the telescope and detectors must be corrected thoroughly using images of stars within the survey field. This in turn imposes stringent limits (requirements) on the allowable rate of change of the PSF. A previous analysis of the SNAP WL systematics (Rhodes et $\mathrm{al}^{2}$ ) simulated the SNAP telescope and detector images, and identified the effects of secondary mirror misalignment, focusing, detector diffusion, diffraction, and variations in PSF owing to structural dry-out shrinkage. It has been shown by a number of researchers (Kaiser ${ }^{3}$, Bernstein ${ }^{4}$ et al) that a given image can be decomposed into its circularly symmetric and asymmetric components. In this paper, we concentrate on asymmetric PSF contributors that are caused by thermal variations, and therefore do not include detector diffusion or pupil diffraction which are both likely to be constant over time. We use a dynamic observatory thermal model to predict quantitative PSF changes during nominal survey attitude maneuvers.

The SNAP observatory (Figure 1) consists of a science instrument and a compact 3-axis stabilized spacecraft. For discussions of the mission architecture, the reader is referred to ${ }^{5,6,7,8}$. The instrument consists of a $2 \mathrm{~m}$ diameter, widefield three-mirror anastigmat (TMA) imaging system ${ }^{9,10,11,12}$, stray light baffle, a focal plane with 36 visible, and 36 near infrared (NIR) detectors ${ }^{13}$ and an integral field unit spectrograph ${ }^{14}$. The focal plane is passively cooled to $140 \mathrm{~K}$ in order to reduce dark noise in the detectors. Observatory commissioning occurs during the first three months after launch. The next 18 months will be used for construction of a Hubble diagram based on discovery of $\sim 2000$ supernovae, and a deep complimentary WL survey. A wide-field (1000 square degree) gravitational weak lensing survey will begin 21 months after launch. 


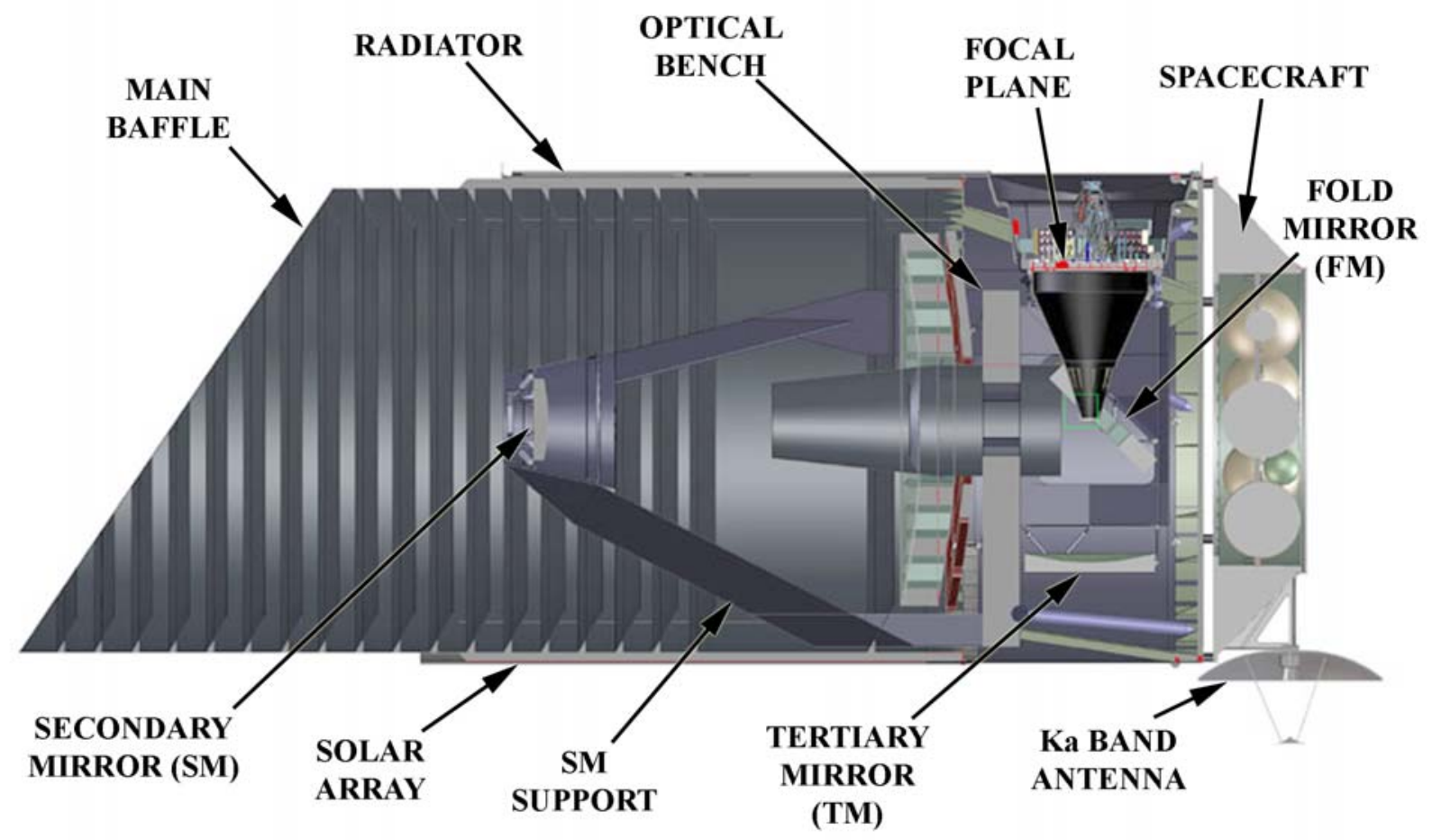

Figure 1: SNAP observatory cross-section

The dark astronomical survey zones are near the ecliptic poles, which make possible an observatory layout that uses a fixed solar array and a fixed passive radiator, both of which help keep the observatory highly rigid with normal mode resonances all lying above $10 \mathrm{~Hz}$. The planned L2 orbit location, free of eclipses and Earth albedo, provides a benign thermal environment. The combination of a rigid observatory and a nearly constant thermal environment is expected to deliver point-spread functions (PSFs) that are significantly more stable than PSFs from other space observatories, e.g. HST. (For a discussion of HST see for example Rhodes et $\mathrm{al}^{15}$.) Solar heating of the telescope is blocked by the main outer baffle which carries the solar array and is otherwise covered by multi-layer insulation (MLI). The outer baffle is not coupled mechanically to the telescope; it mounts directly to a ring at the spacecraft interface (see Figure 2). The telescope mounts to the ring via a semi-kinematic bipod mount. Telescope mirrors are expected to be made of ultra-low expansion glass such as Corning ULE or Schott Zerodur with a coefficient of thermal expansion (CTE) between 10 and 30 parts per billion per kelvin ( $\mathrm{PPB} / \mathrm{K})$. The telescope structure is made of M55J (or similar) composites (-220 PPB/K). A 5 degree of freedom secondary mirror support allows for mirror alignment during ground testing, initial alignment onorbit, and periodic (every 6 months) realignment to correct for any long-term dryout or creep effects in the structure. For the 0.4 to $1.7 \mu \mathrm{m}$ SNAP survey band, thermal self-emissions do not preclude the use of ambient temperature optics, and such are baselined for the SNAP optical system. 


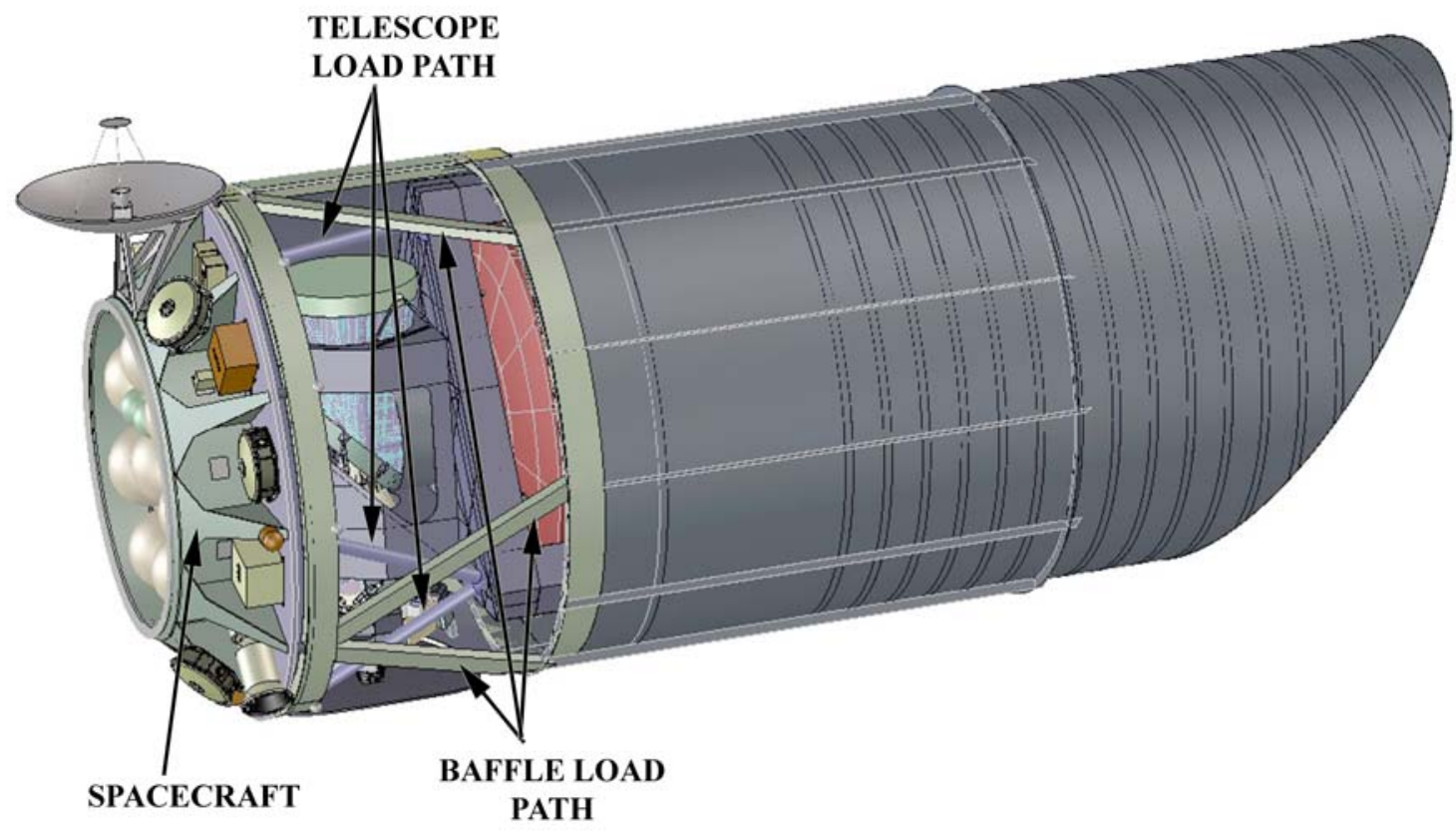

Figure 2: Main optical baffle and telescope structure attach directly to a ring located at the spacecraft interface. This mechanically decouples thermally-induced distortions in the main baffle from the telescope structure.

The baseline optical configuration is designated TMA- 65 , and has a focal length of $21.66 \mathrm{~m}$. The overall length of the telescope is $3 \mathrm{~m}$, which leads to a telephoto advantage of 7 . In order to build such a compact telescope, one requires a fast (f/1) system between the primary and secondary mirrors (PM and SM). As will be shown later in this study, the system is most sensitive to misalignment between the PM and SM. In order to stabilize this structure, a network of thermal sensors and heaters will be employed throughout, in particular on the SM supports. This thermal control system is non-challenging, and well within state of the art at the time of this writing. Predictions of the thermo-optical performance of the thermal control system are modeled and presented below.

\section{IDEAL TELESCOPE PERFORMANCE PREDICTIONS}

The optical design for the SNAP observatory, TMA-65, consists of a concave $\varnothing 2 \mathrm{~m}$ prolate ellipsoid primary mirror, convex hyperbolic secondary mirror, flat folding mirror, concave prolate ellipsoid tertiary mirror and a flat annular focal surface. The PSF and therefore the optical performance are not constant across the focal plane. Figure 3 shows rms WFE for TMA-65 as a function of off-axis angle. This is the design residual error, and indicates the theoretical performance limit of TMA-65. Figure 4 shows the geometric limit of spot sizes for TMA-65, and Figure 5 shows sample PSFs for eight angles spanning the FOV. 


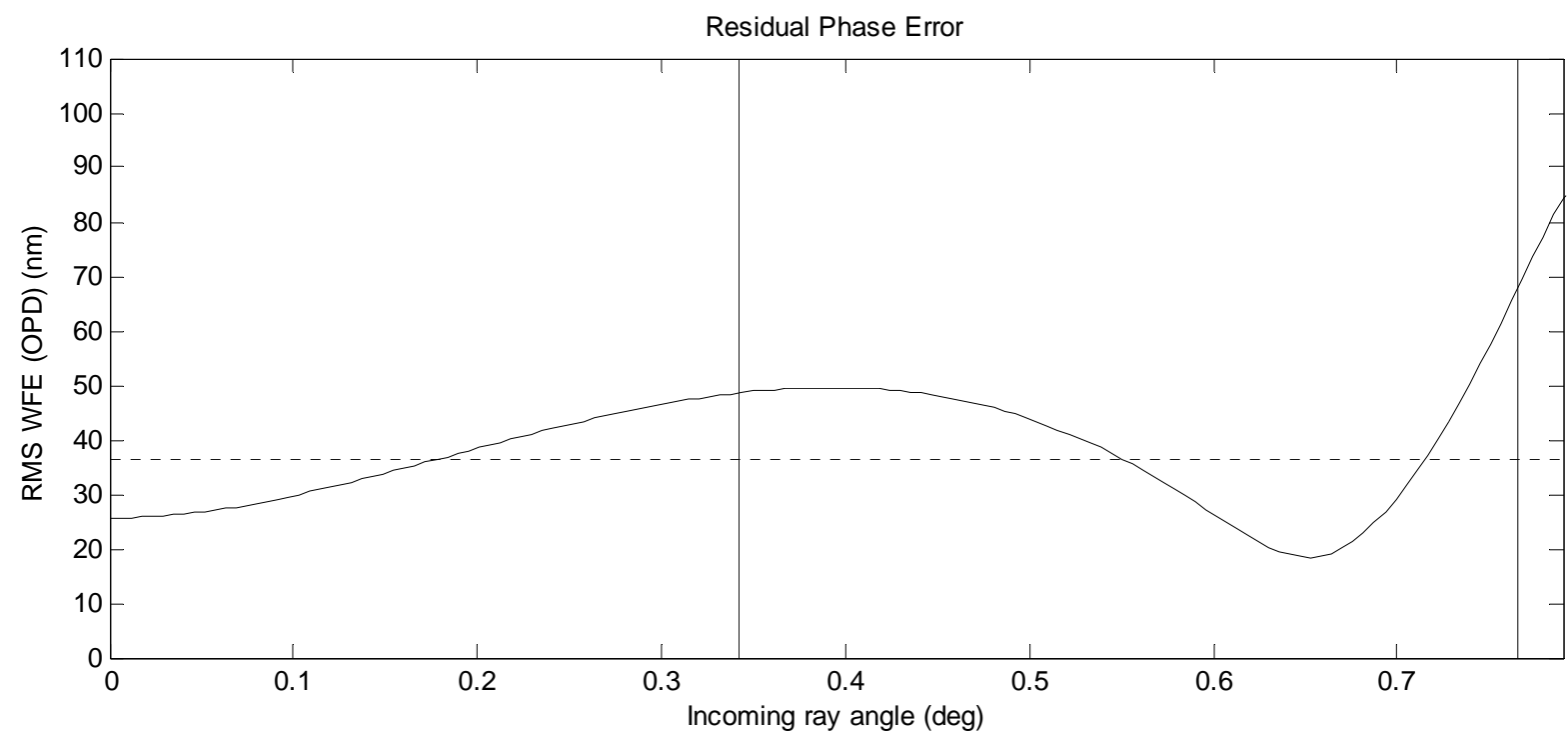

Figure 3: Perfect telescope residual error as a function of off-axis angle. Vertical lines indicate the limit of the FOV. The horizontal, dashed line indicates the pixel-weighted average WFE, $36.8 \mathrm{~nm}$.

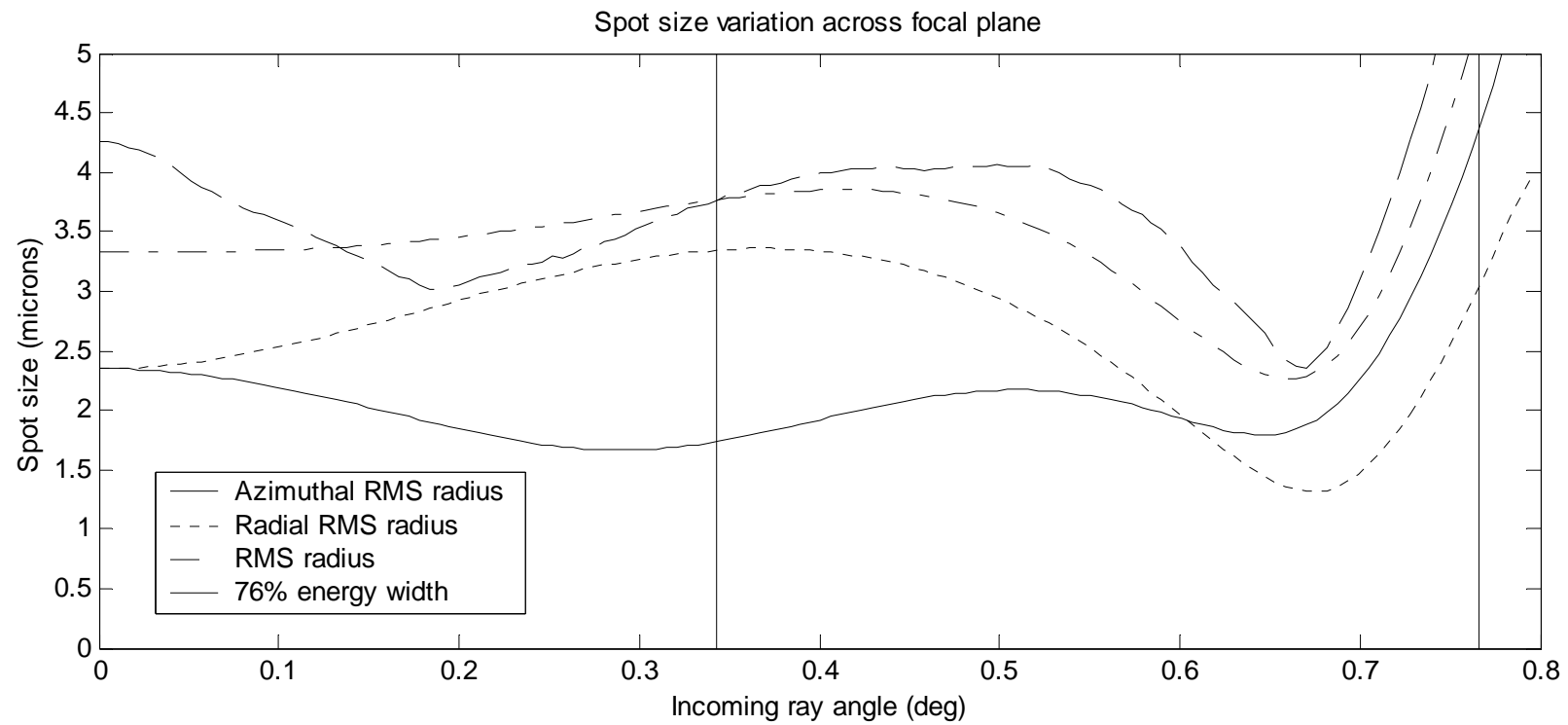

Figure 4: rms spot size, and $76 \%$ energy width as a function of off-axis angle. Best optical performance is at $\sim 0.65^{\circ}$ off-axis, and performance degrades rapidly near the outer edge of the field of view. Vertical lines indicate the limit of the FOV. 

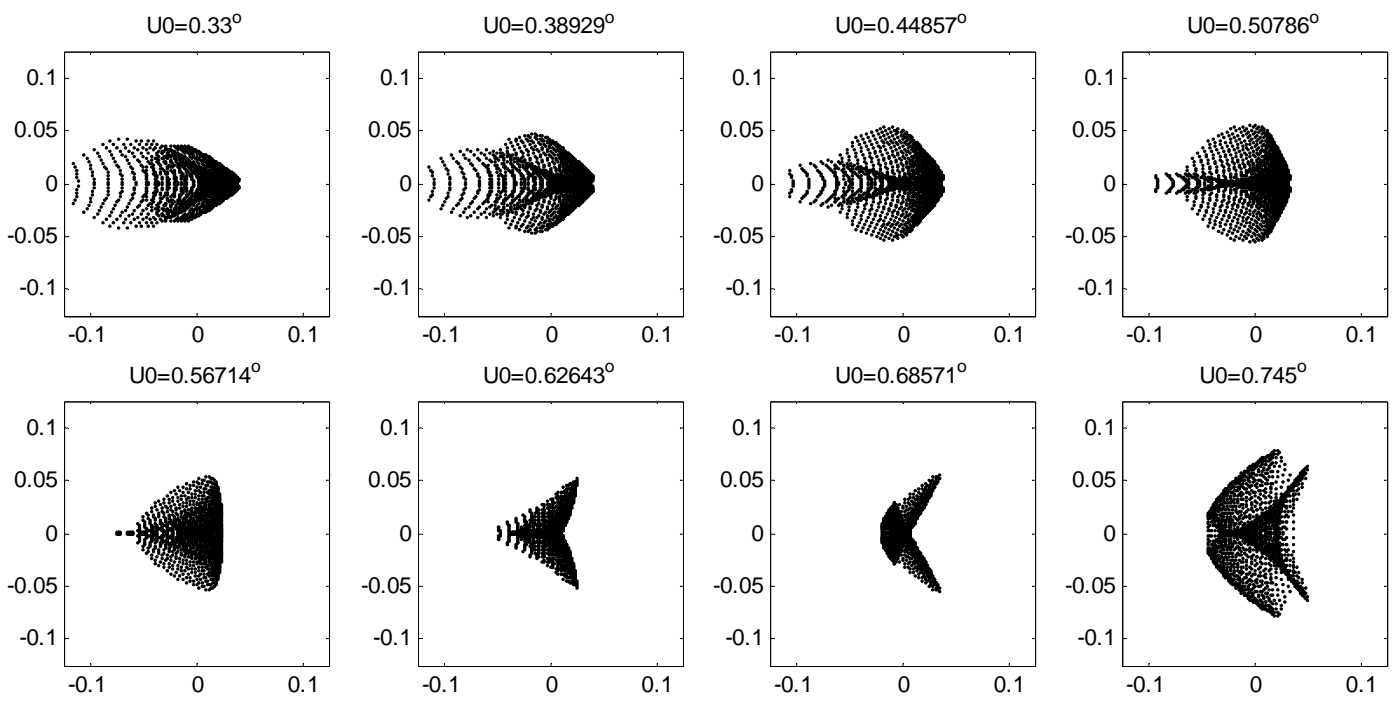

Figure 5: Geometric spot diagrams for select off-axis angles. Both axes are arcsec. Note, near inner radius of the FOV, the spot has a radial bias (horizontal in this figure), while near the extremes of the FOV, the spot has a qualitatively more tangential bias (vertical in this figure).

TMA-65 is diffraction limited longward of $\lambda=1 \mu \mathrm{m}$. Its mean rms geometric spot size is $2.3 \mu \mathrm{m}$, while the diffraction rms size is $4 \mu \mathrm{m}$. The typical range of expected galaxy sizes is $0.1-0.2$ arcsec rms radius, or $10.5-21 \mu \mathrm{m}$ on the focal plane. Because of this, the ellipticity or anisotropy computed from the irradiance profile is typically smaller than that computed from the geometric blur pattern alone. For this study, we wish to quantify the various contributions to the anisotropy in a way that avoids complications arising from choice of wavelength and target size, and therefore base our fractional ellipticity and anisotropy calculations on the diffraction-free geometric blur spot size. One should note that diffraction (not considered in this study) will circularize the PSF, and reduce ellipticity significantly.

\section{WEAK-LENSING PSF STABILITY METRICS}

The SNAP weak-lensing program will map foreground dark matter concentrations by measuring correlated shear patterns in the images of background galaxies. Shear introduced by the optical system, especially a change introduced over short timescales, masks the desired galactic shear signal. Combinations of Gaussian-weighted moments of the PSF are used to quantify both the ellipticity introduced by the telescope, and the weak-lensing shear signal. Commonly used combinations (metrics) include ellipticity, anisotropy, effective anisotropy and whisker.

Weighted moments of the PSF are defined:

$$
\begin{gathered}
M \equiv \int P(x, y) W(x, y) d x d y \\
\bar{x} \equiv(1 / M) \int x P(x, y) W(x, y) d x d y \\
\bar{y} \equiv(1 / M) \int y P(x, y) W(x, y) d x d y \\
P_{x x} \equiv(1 / M) \int(x-\bar{x})^{2} P(x, y) W(x, y) d x d y \\
P_{y y} \equiv(1 / M) \int(y-\bar{y})^{2} P(x, y) W(x, y) d x d y \\
P_{x y} \equiv(1 / M) \int(x-\bar{x})(y-\bar{y}) P(x, y) W(x, y) d x d y
\end{gathered}
$$

and the weight factor $W$ is defined: 


$$
W \equiv \exp \left(-\frac{x^{2}+y^{2}}{2 \sigma^{2}}\right)
$$

Unweighted moments $(\mathrm{W}=1)$ converge slowly, and tend to diverge in the presence of detector noise. To increase the $\mathrm{S} / \mathrm{N}$ of the shape measurement, one uses an a-priori estimate of the galaxy size to select a Gaussian weighting factor when performing moment integration. This factor emphasizes light within the core of the galaxy, and drops to zero exponentially with increasing distance from the core.

One may define polarization, or ellipticity from these weighted moments as

$$
\begin{gathered}
e_{1} \equiv \frac{P_{x x}-P_{y y}}{P_{x x}+P_{y y}} \\
e_{2} \equiv \frac{2 P_{x y}}{P_{x x}+P_{y y}} \\
\sigma_{*}^{2} \equiv \frac{P_{x x}+P_{y y}}{2}=\frac{P_{\text {total }}}{2}
\end{gathered}
$$

Qualitatively, $e_{I}$ indicates alignment with the coordinate axes. A positive $e_{l}$ indicates an elliptical shape oriented along the $\mathrm{X}$ axis, while a negative $e_{1}$ implies alignment with the $\mathrm{Y}$ axis. Ellipticity $e_{2}$ indicates diagonal orientation, and the sign of $e_{2}$ corresponds to the sign of the slope, in the XY plane. Orientation of the ellipticity is determined from the arctangent of the ratio $e_{1} / e_{2}$. See Kaiser ${ }^{3}$ for a helpful graphic illustrating ellipticity magnitude and orientation as a function of $\mathrm{e}_{1} \mathrm{e}_{2}$.

The length scale of the ellipticity may be written in dimensional (called whisker, $S$ ), and non-dimensional (anisotropy, $\chi$ ) form as follows:

$$
\begin{gathered}
S(\sigma)=\left(\left(P_{x x}-P_{y y}\right)^{2}+4 P_{x y}^{2}\right)^{\frac{1}{4}} \\
\chi(\sigma)=\frac{(S(\sigma))^{2}}{P_{x x}+P_{y y}}
\end{gathered}
$$

Casertano ${ }^{16}$ defines the effective anisotropy $\chi_{\text {eff }}$ for a circular galaxy of Gaussian size $\sigma$, when imaged in the presence of shear, as: $\chi_{\text {eff }} \equiv \chi(\sigma) P_{\text {total }}(\sigma) /\left[2 \sigma^{2}+P_{\text {total }}(\sigma)\right]$. Effective anisotropy $\chi_{\text {eff }}$ reduces the influence of telescope anisotropy when the $I_{\text {total }}$ (the telescope PSF) is small compared to the size of the observed galaxy $(\sigma)$.

Figure 6 shows the distribution of ellipticity for a perfectly built and aligned TMA-65. For a system without noise, shear signals increase slowly as the weighting scale $\sigma$ is increased. Typical galaxy sizes within the redshift range of SNAP are 0.1-0.2 arcsec, and $\chi_{\text {eff }}$ decreases rapidly with increasing $\sigma$. A weighting factor near the bottom of the range of possible galaxy sizes, $\sigma=0.1$ arcsec, was chosen conservatively to produce the largest $\chi_{\text {eff }}$ (minimum dilution by galaxy size).

Both whisker length and anisotropy are invariant under rotation. As shown in Figure 6, the orientation of the ellipticity changes from radial to azimuthal at an off-axis angle of roughly $0.65^{\circ}$. Due to the aforementioned invariance, and the sensitivity of the WL shear measurement to PSF orientation, an additional metric is necessary to quantify orientation changes to whisker and anisotropy. If Pxx-Pyy and $2 P x y$ are treated as orthogonal coordinate directions, the distorted and undistorted ellipticity components may be subtracted vectorally, to produce an orientation-dependent change in whisker and effective anisotropy $\left(\Delta S\right.$ and $\left.\Delta \chi_{\text {eff }}\right)$. In the relations shown below, a primed term indicates a moment computed from a distorted telescope spot. In sections to follow, these metrics are used to quantify telescope-induced shear contributions. 


$$
\begin{gathered}
\Delta S(\sigma) \equiv\left(\left(\left(P_{x x}-P_{y y}\right)-\left(P_{x x}^{\prime}-P_{y y}^{\prime}\right)\right)^{2}+4\left(P_{x y}-P_{x y}^{\prime}\right)^{2}\right)^{\frac{1}{4}} \\
\Delta \chi(\sigma) \equiv \frac{(\Delta S(\sigma))^{2}}{P_{x x}+P_{y y}} \\
\Delta \chi_{\text {eff }} \equiv \Delta \chi(\sigma) P_{\text {total }}(\sigma) /\left[2 \sigma^{2}+P_{\text {total }}(\sigma)\right]
\end{gathered}
$$

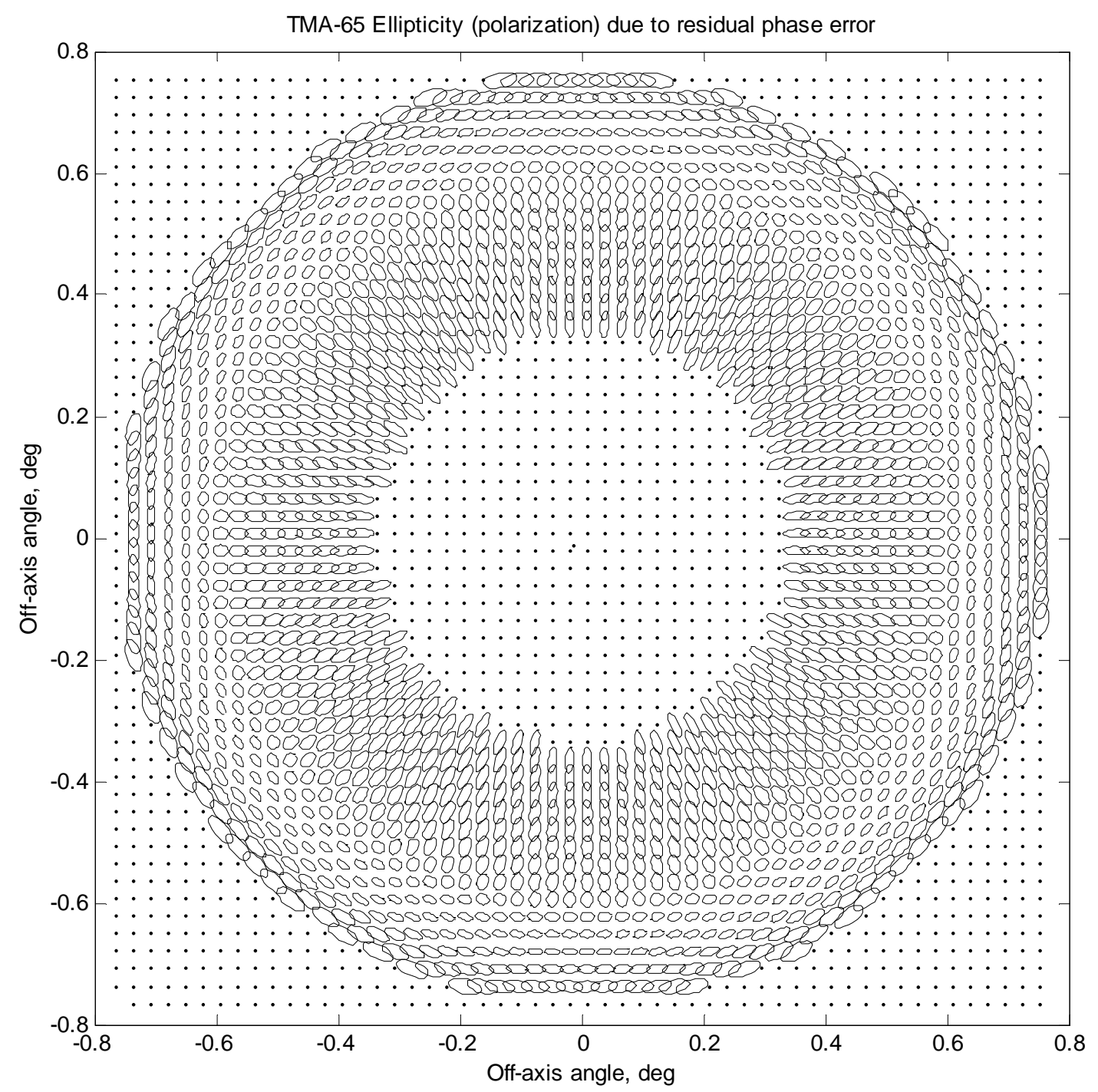

Figure 6: Ellipticity (polarization) of perfectly-built TMA-65. Ellipses shown are second moments, magnified by a factor of 10,000 for the purpose of visualization. Note the strong radial component for angles $<\sim 0.6^{\circ}$, and azimuthal component for angles beyond. The maximum fixed whisker length is 29 milli arcsec, at the outer extreme of the FOV. The reader is urged to examine this figure, along with figure 5 , for a qualitative understanding of the radial ellipticity distribution.

\section{STABILITY REQUIREMENTS \& EFFECTS ON ANISOTROPY}

Extensive work on WL field calibration has been conducted for mountaintop observing programs and for HST studies (e.g Bernstein et $\mathrm{al}^{4}$ and references therein). Briefly, every field exposure contains both stars and galaxies. The stars have essentially no spatial extent. They serve to monitor the instrumental seeing PSF at their locations within 
the field. Stars of suitable magnitude range can be reliably distinguished from galaxies by their image radii. A 2-D PSF interpolating polynomial is constructed from these stars, and evaluated at the field location of each galaxy of interest, to obtain an estimate of the instrumental seeing for that galaxy. The observed anisotropy of each galaxy is then corrected using the instrumental PSF estimate. A specification on telescope PSF and PSF rate of change can be written once a comprehensive budget is constructed that encompasses the several kinds of contributions to the instrumental PSF signature (attitude control system, structural vibrations, thermal, composite structure dryout, etc), and once simulations have been run that include the appropriate statistical density of monitor stars. This present study shows the sensitivity of a baseline telescope system to anticipated thermal transient and composite dryout effects, and will help to guide the specification process.

Requirements for the SNAP supernova program call for a telescope with diffraction limited performance at $1 \mu \mathrm{m}$. Toward this end, a comprehensive WFE budget for the observatory was developed, and error allocations parceled out to various contributors. Of particular interest in this study is the alignment drift section of the WFE budget (see Table 1). Telescope mirrors must be held to the mechanical tolerances listed in the table for the roughly six month intervals between SM refocus. Tolerances listed in the table, while not trivial to achieve, are well within the capabilities of an M55J composite metering structure with temperatures regulated via heaters and thermal sensors.

Weak lensing adds even more stringent requirements for stability of the telescope. For 0.1 arcsec (rms) galaxies, one desires ellipticity predictions with an accuracy of $0.1 \%$ (divide this value by $\sim 3$, for margin). Multiplying $0.03 \%$ by the square of the nominal galaxy size $\left(0.1 \operatorname{arcsec}^{2}\right)$ leads to a target maximum whisker $\Delta \mathrm{S}$ goal of $\sim 2$ milli-arcsec. Changes in the ellipticity distribution are investigated by introducing the mechanical tolerances listed in Table 1 to the position of the optics, raytracing, and comparing the ellipticities to those computed in the pristine case. The most critical tolerances on the system constrain the relative position of the PM and SM. Table 1 also lists the maximum effective anisotropy, maximum whisker and the change induced in both by motion of an optic to its tolerance limit. Effective anisotropies are calculated for a 0.1 arcsec galaxy, and will of course be lower when diluted by a larger galaxy. When the rather large effects of diffraction are considered, the whisker length (and therefore effective anisotropy) changes will be smaller than shown in this study: a purely geometric analysis. Table 1 shows the unsurprising result that WL constraints demand more structural stability than the supernova program. As will be shown in later sections, thermo-mechanical analysis of the observatory predicts much lower changes than the supernova program requirements listed in this table. The effects of composites dryout are constrained quantifying and predicting the observatory dryout rate early in the mission, use of foreground stars to measure telescope-induced ellipticity and by limiting the duration of WL measurements.

\begin{tabular}{|c|c|c|c|c|c|c|c|c|}
\hline \multicolumn{9}{|c|}{ TMA-65 Alignment Drift Budget } \\
\hline Mirror & Misalignment & $\begin{array}{l}\text { OPD (nm } \\
\text { RMS) }\end{array}$ & $\begin{array}{c}\text { Drift } \\
\text { Tolerance }\end{array}$ & Units & $\begin{array}{c}\text { Max Whisker S } \\
\text { (milli arcsec) }\end{array}$ & $\begin{array}{l}\text { Max. } \Delta S \text { (milli } \\
\text { arcsec) }\end{array}$ & Max. Xeff (\%) & $\begin{array}{c}\text { Max. } \Delta \text { Xeff } \\
(\%)\end{array}$ \\
\hline Pristine & None & & & & 29 & 0.0 & 3.8 & 0.00 \\
\hline \multirow{3}{*}{ Primary } & Despace & 2.24 & 0.1 & $\mu \mathrm{m}$ & 28 & 6.3 & 3.6 & 0.18 \\
\hline & Decenter & 3.15 & 0.5 & $\mu \mathrm{m}$ & 29 & 10 & 3.8 & 0.44 \\
\hline & Tilt & 3.12 & 0.2 & $\mu \mathrm{rad}$ & 29 & 9.9 & 3.8 & 0.44 \\
\hline \multirow{3}{*}{ Secondary } & Despace & 5.70 & 0.1 & $\mu \mathrm{m}$ & 27 & 11 & 3.2 & 0.57 \\
\hline & Decenter & 3.15 & 0.3 & $\mu \mathrm{m}$ & 29 & 2.2 & 3.8 & 0.02 \\
\hline & Tilt & 0.66 & 0.2 & $\mu \mathrm{rad}$ & 26 & 14 & 3.0 & 0.84 \\
\hline \multirow{2}{*}{ Fold } & Despace & 2.00 & 1 & $\mu \mathrm{m}$ & 28 & 8.9 & 3.5 & 0.35 \\
\hline & Tilt & 1.36 & 2 & $\mu \mathrm{rad}$ & 29 & 4.1 & 3.8 & 0.08 \\
\hline \multirow{3}{*}{ Tertiary } & Despace & 2.50 & 1 & $\mu \mathrm{m}$ & 26 & 13 & 3.1 & 0.72 \\
\hline & Decenter & 2.20 & 10 & $\mu \mathrm{m}$ & 26 & 15 & 3.1 & 0.97 \\
\hline & Tilt & 1.70 & 5 & $\mu \mathrm{rad}$ & 26 & 14 & 3.0 & 0.83 \\
\hline
\end{tabular}

Table 1: Alignment drifts allowable for the supernova program, over a six month period, are listed both in mechanical alignment drift units and pupil coordinates (nm rms wavefront error). The maximum (anywhere in the FOV) whisker length (milli-arcsec), effective anisotropy (\%) and changes to both are shown. 


\section{PREDICTED THERMAL ENVIRONMENT \& ANISOTROPY}

Due to the sensitivity of the telescope to motion of the SM, an estimate of the stability of the SM position and orientation to temperature changes is of key importance. The L2 orbit is one of the most stable locations to place an observatory, and the mission is designed to preclude passage through Earth's shadow. Consequently, the overall solar flux varies slowly, due to seasonal changes, throughout the mission. A large number of test cases have been analyzed, and three that introduce the largest thermal variations identified. These include:

1. Seasonal variations in distance from the Sun, as the observatory orbits at $L 2$, which will result in $1 / \mathrm{R}^{2}$ variations of the solar flux $\left(1298 \mathrm{~W} / \mathrm{m}^{2}\right.$ to $\left.1384 \mathrm{~W} / \mathrm{m}^{2}\right)$.

2. Mission design calls for $90^{\circ}$ rolls of the observatory every three months. These will change the solar flux loading distribution on the observatory.

3. Daily slews to point the body-mounted antenna toward Earth will change the thermal environment.

To address these test cases, and quantify induced anisotropy, a combined thermal, structural, optical analysis of the observatory was undertaken. A lumped parameter finite difference model of the observatory was developed, and a thermal control system modeled on the SM support members. This system included 247 nodes, and 37 heater/thermistor pairs (see Figures $7 a$ and $7 b$ ). A proportional controller was modeled, and thermal excursions of the individual legs under the three test cases computed.

A finite element model (FEM) of the telescope secondary support struts was developed, and the motion of the SM due to a small $(1 \mu \mathrm{m})$ expansion of one of the legs computed. Despace, decenter and tilt of the SM were found to be $0.38 \mu \mathrm{m},-$ $0.95 \mu \mathrm{m}$ and $-1.1 \mu \mathrm{rad}$, respectively for a $1 \mu \mathrm{m}$ SM support leg expansion. (See Figure $7 c$ for a view of the FEM, with thermal distortions amplified by a factor of 250,000 .)

Worst-case temperature changes were computed, and converted to SM support strut expansion using the negative 220 $\mathrm{PPB} / \mathrm{K}$ CTE of M55J composites. Strut expansions are converted to mirror motion by scaling the results of the FEM analysis to the thermal predictions, and maximum effective anisotropies $\chi_{\mathrm{eff}}$ are computed relative to the pristine case. These changes are listed in Table 2.

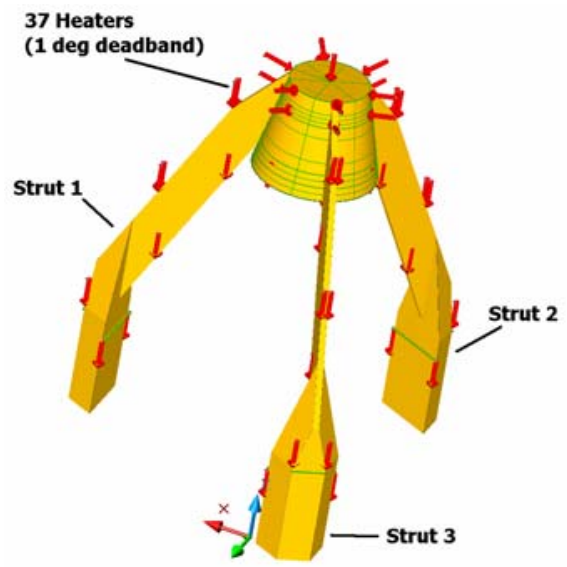

a.

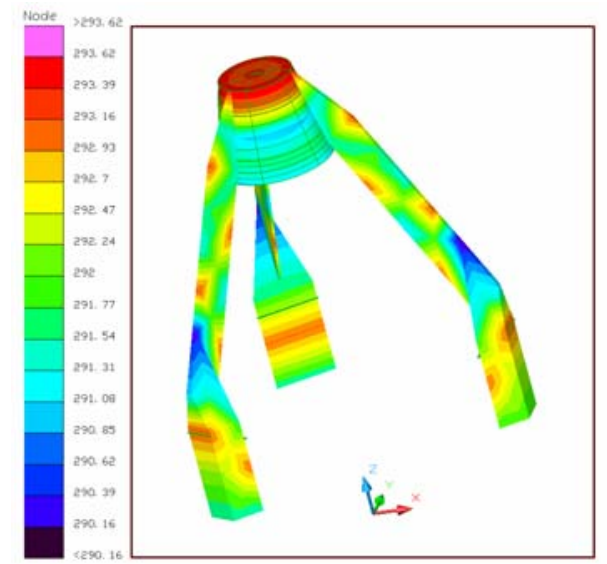

b.

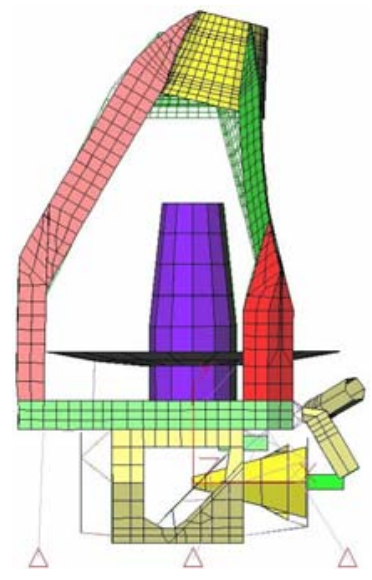

c.

Figure 7: A lumped-parameter finite difference thermal model of the SM support structure is shown in $\boldsymbol{a}$ and $\boldsymbol{b}$. A thermal control algorithm using 37 heater/thermistor pairs and proportional control is simulated in the model. Cases studied include $90^{\circ}$ roll, communication slew and seasonal solar flux variation. A FEM of SM tripod with one leg expanded by $1 \mu \mathrm{m}$ is shown in $c$ (expansion is exaggerated by a factor of 250,000 to allow visualization of the thermal deformation). Results from the thermal and FEM analyses are used to predict transient mirror motion, and consequent ellipticity and anisotropy. 


\begin{tabular}{|l|l|c|c|c|}
\hline Event & Note & $\begin{array}{c}\text { Leg } \Delta l \\
(\mathrm{~nm})\end{array}$ & $\begin{array}{c}\text { Max. } \Delta \mathrm{S} \\
(\mu \operatorname{arcsec})\end{array}$ & Max $\Delta \chi_{\text {eff }}$ change $(\%)$ \\
\hline $90^{\circ}$ roll (every 3 months) & View to sun changes & 15.0 & 181 & 0.010 \\
\hline Communication slew & View to sun changes & 10.0 & 120 & 0.007 \\
\hline $\begin{array}{l}\text { Seasonal } 1 / \mathrm{R}^{2} \text { variations } \\
\text { in solar flux }\end{array}$ & $\begin{array}{l}1298 \mathrm{~W} / \mathrm{m}^{2} \text { minimum } \\
1384 \mathrm{~W} / \mathrm{m}^{2} \text { maximum }\end{array}$ & 8.0 & 96 & 0.005 \\
\hline
\end{tabular}

Table 2: Predictions from thermal, structural and optical analysis for maximum SM support leg expansion, whisker length change and change in effective anisotropy. Recall from Section 4, the goal for maximum $\Delta \mathrm{S}$ is 2 milli-arcsec. Thermal stability predictions are for timescales greater than one month.

\section{COMPOSITE STRUCTURE DRYOUT AND SHRINKAGE}

Although we have not yet formulated a quantitative model of the structural shrinkage arising from on-orbit dryout, it is clear from examination of the refocused position of the HST secondary mirror ${ }^{17}$ that the HST metering structure has shrunk by 150 microns over its first eight years of operation. For SNAP, shrinkage effects are exacerbated by the fast primary mirror compared with HST. Because PSF anisotropy changes are significant with submicron alterations in structural lengths, we anticipate that a comprehensive PSF budget will need to include this effect.

Dryout is expected to decrease the PM/SM spacing as the mission progresses. Figure 8 shows ellipticity changes on one quadrant of the focal plane introduced by $1 \mu \mathrm{m}$ dryout shrinkage of the PM-SM support structure. Ellipse geometries are exaggerated by a factor of 10,000, and represent the ellipticity variations typical in the first months after launch.

Advances in the state of the art of graphite fiber cyanate ester laminates have reduced the moisture retention level to $<0.2 \%$ by weight ${ }^{18}$ for a laminate with a coefficient of moisture expansion (CME) of $<200$ parts per million (PPM) $/ \% \mathrm{H}_{2} 0$. The TMA-65 PM and SM are separated by $2 \mathrm{~m}$, roughly half the separation of the PM and SM on the Hubble Space Telescope (HST). These factors suggest that the SNAP telescope shrinkage will be of the order of 75 microns, which is significant for the SNAP PSF, and will require refocusing.

Based on the SM despace numbers in Table 1, and the $\sim 2$ milli-arcsec whisker change ceiling mentioned in Section 4, one derives a maximum SM/PM despace variation of 150-250nm over a set of WL measurements (for example, 24 hours between antenna slews). The timescale over which composite dryout shrinkage exceeds this level will lengthen as the mission progresses, with the majority of shrinkage expected to occur during observatory commissioning en-route to L2 (3 months). Periodic refocusing will be necessary as the structure shrinks. In the simplest desorption model, the moisture content decays exponentially, with a time constant determined by resin composition and layup thickness. On SNAP, dryout $1 / \mathrm{e}$ timescales are shortened to $\sim 1$ month by specifying a maximum $2 \mathrm{~mm}$ wall thickness throughout the structure $^{19}$. The baseline mission plan has photometric and deep WL surveys beginning about 3 months after launch. During this phase, the focused position of SM will be tracked, and the ellipticity induced by the telescope predicted using the SM position, and foreground stars. A dedicated 1000 square degree WL survey begins 21 months after launch. Although we have not yet modeled the dryout process, if the exponential curve is appropriate, 21 months after launch the composite structures on SNAP are computed to have sufficiently small per-day length changes to meet expected whisker change requirements. 


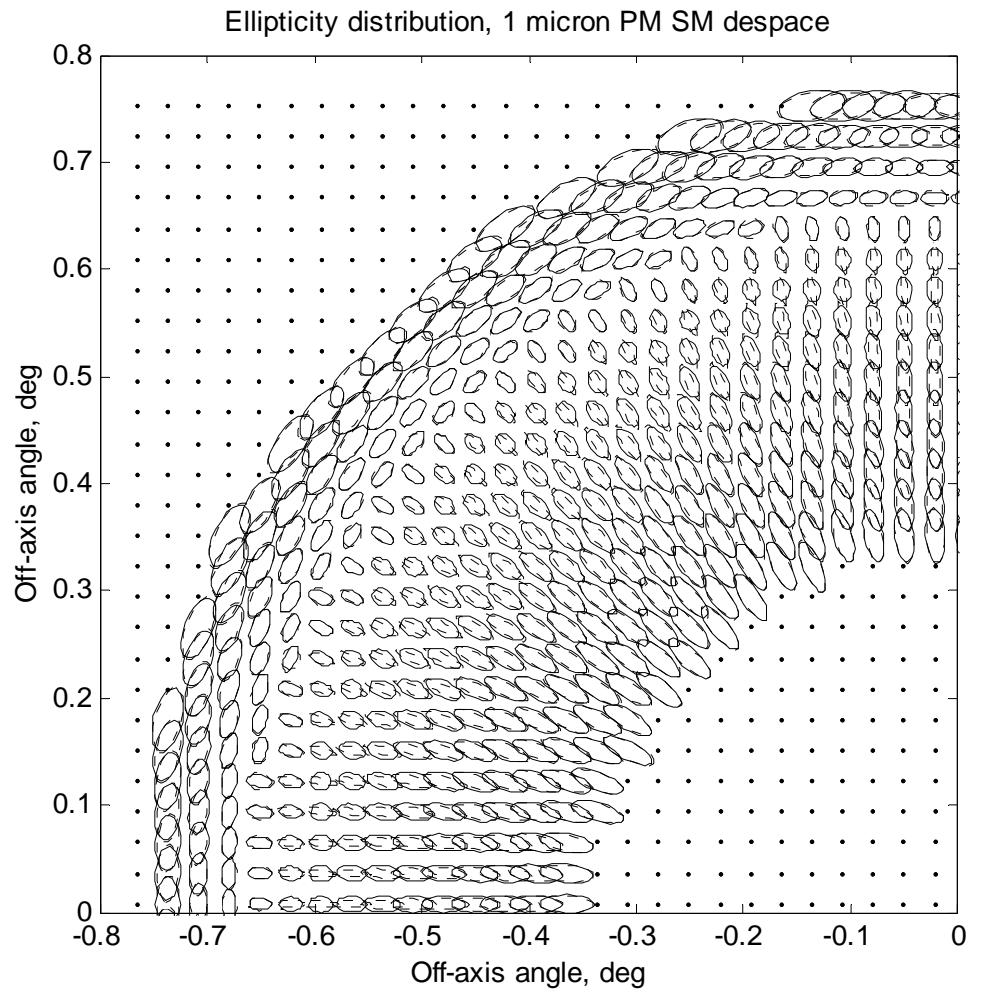

Figure 8: Ellipticity (second moment) distribution change introduced by $1 \mu \mathrm{m}$ PM-SM despace are shown as dashed lines (solid ellipses are those produced by a perfect telescope). In this figure, spot sizes are scaled by a factor of 10,000 relative to the plate scale, for the purpose of visualization. A photometric and deep WL survey begins 3 months after launch. During this time, the SMmeasured dryout rate, along with foreground stars will be used to predict the telescope ellipticity contribution. Wide-field WL observations will begin 21 months after launch, at which point composite structures on SNAP are expected to be nearly dry. Based on an exponential dryout model, and a one month 1/e composite structure dryout timescale, the observatory is predicted to have a sufficiently small SM-PM despace shrinkage for a WL survey.

\section{CONCLUSIONS}

A combined thermal, structural and optical analysis of the SNAP observatory was performed. Point spread function metrics relevant to the SNAP weak lensing survey were discussed, and the ability to quantify whisker length and effective anisotropy for a misaligned TMA-65 optical configuration developed. Changes in these metrics for optic motions to the extremes of the alignment drift budget were quantified (without the effects of diffraction). Predictions for worst-case thermal cases $\left(90^{\circ}\right.$ roll, seasonal flux variations and antenna slew) and composites dryout were investigated using the thermo-structural-optics code. Changes to whisker length and effective anisotropy due to thermal transients were found to be very small compared to stability requirements for WL shear measurement. Variations in whisker and effective anisotropy due to composite dryout were investigated, and are found to be acceptably small for daily WL surveys beginning 21 months after launch.

\section{ACKNOWLEDGMENTS}

The authors gratefully acknowledge useful discussions with Prof. Gary Bernstein. This work was supported by the Director, Office of Science, of the U.S. Department of Energy under Contract No. DE-AC03-76SF00098. 


\section{REFERENCES}

1. G. Aldering, et al, Supernova/Acceleration Probe: a Satellite Experiment to Study the Nature of the Dark Energy, astro-ph/0405232, 2004.

2. J. Rhodes et al, Astroparticle Phys. v.20 377-389, 2004.

3. Kaiser et al, ApJ v.449, 460-475, 1995.

4. G. Bernstein et al, AJ v.123, 583-618, 2002.

5. S. Perlmutter et al, Astrophys. J., 517, p.565, 1999.

6. A.G. Riess et al, Astron. J., 116, p.1009. 1998.

7. G. Aldering et al, Proc. SPIE $4835 \# 21,2002$.

8. A. Kim et al, Proc. SPIE $\mathbf{4 8 3 6}$ \#10, 2002.

9. M. Lampton et al, Proc. SPIE 4849 pp.215-226, 2002.

10. M. Lampton et al, Proc. SPIE 4854, 2002.

11. M. Lampton et al, Proc. SPIE 5166, 2003.

12. M. Sholl et al, Proc. SPIE 5487, 2004.

13. C. Bebek et al, Proc. SPIE 5164 \#10, 2003.

14. A. Ealet et al, Proc. SPIE $4850 \# 165,2002$.

15. J. Rhodes et al, ApJ v.536, 79-100, 2000

16. S. Casertano, Some Considerations on PSF Asymmetry and its Impact on the Measurement of Galaxy Shapes, STScI-JWST-R-2002-0003, 2003.

17. http://www.stsci.edu/hst/observatory/focus/focushistory.html

18. C. Blair et al, Proc. SPIE 1690 300-308, 2002.

19. Private communication, Composite Optics Incorporated, 2002. 